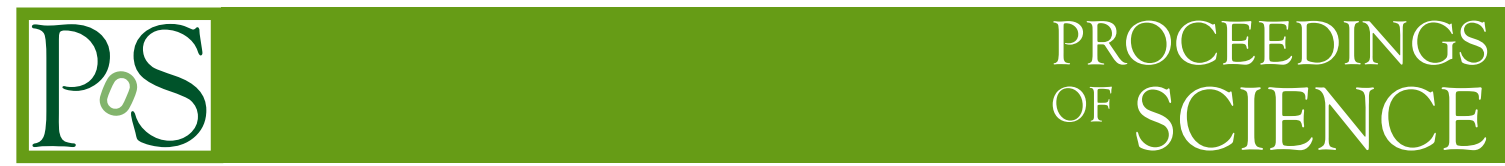

\title{
Flavor Physics with CMS: status and perspectives
}

\author{
Urs Langenegger* \\ Paul Scherrer Institute \\ E-mail: urs.langenegger@psi.ch
}

The Compact Muon Solenoid (CMS) experiment at the Large Hadron Collider (LHC) at CERN started to collect proton proton collision data in fall 2009. These proceedings provide a flavor of the initial detector performance and early measurements of relevance to heavy flavor physics. The second part discusses the CMS perspective in rare $B_{s}^{0}$ decays and top quark flavor physics. (Un)Fortunately the results presented at this conference have been superseeded already a few weeks after this conference due to the very large increase of the LHC luminosity.

Flavor Physics and CP Violation - FPCP 2010

May 25-29, 2010

Turin, Italy

${ }^{*}$ Speaker. 


\section{Introduction}

The primary focus of heavy flavor physics at the Compact Muon Solenoid (CMS) experiment rests on beauty and top quark decays. The production of these quarks is governed by the strong and electroweak forces and therefore (differential) production cross section measurements provide quantitative tests of these interactions. Only the decays of these quarks (or the hadrons they form) provide access to flavor physics in a more narrow sense. Since the cross sections for top and beauty quark production are expected to be very large at the Large Hadron Collider (LHC), these studies can be performed already initially with small data samples. Next to interesting physics studies, heavy flavor analyses also provide for excellent opportunities in detector and trigger commissioning and understanding.

The initial heavy flavor physics program at CMS will be dominated by beauty decays, where interesting results can be expected already with an integrated luminosity of less than $1 \mathrm{fb}^{-1}$, achievable before the end of 2011. The decays under study include the very rare decay $B_{s}^{0} \rightarrow \mu^{+} \mu^{-}$, $B_{s}^{0} \rightarrow J / \psi \phi$, and $\Lambda_{b} \rightarrow \mu^{+} \mu^{-} \Lambda$ (where flavor tagging without mistagging is possible due to the $\Lambda$ decay kinematics). At later stages, rare top quark decays mediated by flavor-changing neutral currents (FCNC) will open a new field in flavor physics.

\section{The CMS Detector and its Performance}

The CMS experiment was designed to provide the best possible lepton identification, excellent $b / \tau$ tagging, with good jet energy and missing transverse energy measurement. A detailed description of the CMS detector can be found in Ref. [1]. The CMS experiment uses a right-handed coordinate system, with the origin at the nominal proton-proton collision point, the $x$ axis pointing towards the center of the LHC ring, the $y$ axis pointing upwards (perpendicular to the LHC plane), and the $z$ axis pointing along the anticlockwise beam-direction. The polar angle $(\theta)$ is measured from the positive $z$ axis and the azimuthal angle $(\phi)$ is measured from the positive $x$ axis in the $x y$ plane. The radius $(r)$ denotes the distance from the $z$ axis and the pseudorapidity is defined by $\eta=-\ln \tan (\theta / 2)$.

The subdetectors relevant for heavy flavor analyses are the inner tracker, consisting of silicon pixel and silicon strip layers, and the muon stations. The tracker is immersed in a $3.8 \mathrm{~T}$ axial magnetic field. The pixel detector consists of three barrel layers and two end-cap disks at each barrel end. The strip tracker has 10 barrel layers and 12 end-cap disks. The tracker provides excellent momentum and vertexing resolution despite its substantial thickness of up to $1.8 X_{0}$. Muons are measured in gas-ionization detectors embedded in the iron return yoke. In the barrel, there is a drift tube system interspersed with resistive plate chambers, and in the end-caps there is a cathode strip chamber system, also interspersed with resistive plate chambers. The muon detectors provide for a "standalone muon" reconstruction with limited transverse momentum resolution. This is improved in the "global muon" reconstruction, where a standalone muon is linked to a track measured in the silicon tracker. To increase the identification efficiency for low transverse momentum $\left(p_{\perp}\right)$ muons, "tracker muons" are formed by linking tracks in the silicon tracker with hits in the innermost muon detectors (these hits need not be part of a standalone muon). The muon identification efficiency 
was measured in cosmic-ray data to be above $95 \%$ for $p_{\perp}>20 \mathrm{GeV}$ [2]. With more beam data, the muon identification efficiency will be measured with muons from $J / \psi$ and $\Upsilon$ decays.

For heavy flavor physics the most important first-level (L1) trigger is based on the muon system. The high-level trigger combines this with information from the inner tracker. The single-muon trigger efficiency was measured to be larger than $95 \%$ for muons with $p_{\perp}>5 \mathrm{GeV}$. At the very low instantaneous luminosity for the data taking periods covered in this presentation, the triggers were based on beam monitoring devices. Beam scintillator counters and beam pickup timing detectors provide efficient triggers for minimum bias events.

After a long period of cosmic ray data, proton proton collision data taking started in late fall 2009 at center of mass energies $\sqrt{s}=900 \mathrm{GeV}$ and $\sqrt{s}=2.36 \mathrm{TeV}$. On March 30, 2010, the first proton proton collisions at $\sqrt{s}=7 \mathrm{TeV}$ were recorded. The default Monte Carlo (MC) simulation of the proton proton collisions in CMS is based on the PYTHIA program [3] and underestimated the average number of tracks per event as illustrated in Fig. 1(a). The detector performance, however, is simulated very well [4]. Exemplary performance plots of the pixel detector show the cluster charge in Fig. 1(b) and the primary vertex resolutions in Fig. 1(c) and (d).

The long-lived resonances $K_{S}^{0} \rightarrow \pi^{+} \pi^{-}$and $\Lambda \rightarrow p \pi^{-}$are reconstructed from tracks of oppositely charged particles with an impact parameter in the transverse plane of $d_{x y} / \sigma\left(d_{x y}\right)>0.5$ and at least 5 hits. The track pairs are fit to a common vertex which is required to be far from the primary vertex $d_{x y} / \sigma\left(d_{x y}\right)>15$. The masses, resolutions, and lifetimes measured in data are consistent with the world averages and the detector resolution in MC simulation. Fig. 2 illustrates the reconstructed candidates. The MC simulated event sample is normalized to the reconstructed $K_{S}^{0}$ yield. Figure 2(b) indicates that the production of strange baryons is underestimated in the MC simulation.

Combining $\Lambda$ candidates with pions (kaons), the CMS experiment also reconstructed $\Xi^{-}\left(\Omega^{-}\right)$ candidates. Tracks with $p_{\perp}>600 \mathrm{MeV}$ and a three-dimensional impact parameter $d_{0}>3 \sigma$ and with the expected charge correlation to the pion track of the $\Lambda$ candidate were chosen. Clear peaks are visible in Fig. 3 for both cases in events with only one reconstructed $\Xi^{-}$or $\Omega^{-}$candidate.

In the silicon strip tracker, the measurement of specific ionization energy loss allows for the identification of low momentum particles. The inclusive reconstruction of $\phi \rightarrow K^{+} K^{-}$is achieved by requiring for both kaon tracks either $p_{\perp}>1 \mathrm{GeV}$ or $\left|m-m_{K}\right|<200 \mathrm{MeV}$, as obtained from the $d E / d x$ measurement. The production of deuterons is clearly observed in data, but was initially absent in the MC simulation.

\section{Exclusive Charm Signals}

The reconstruction of heavy resonances at CMS started immediately after the first $p p$ collisions at $\sqrt{s}=7 \mathrm{TeV}$. No effort was made to coordinate the active analyses, and therefore the dataset and selection criteria vary considerably among the three analyses presented here [5]. In all cases the peak positions are consistent with the world averages and the peak widths are consistent with the expectation from MC simulation.

For the inclusive reconstruction of the decay $D^{0} \rightarrow K^{-} \pi^{+}$in 27 million minimum bias events the following selection criteria were applied. The transverse momenta were required to be $p_{\perp}\left(\pi^{+}\right)>$ $1.0 \mathrm{GeV}, p_{\perp}\left(K^{-}\right)>1.25 \mathrm{GeV}$, and $p_{\perp}\left(D^{0}\right)>3.0 \mathrm{GeV}$. The distance of closest approach between 


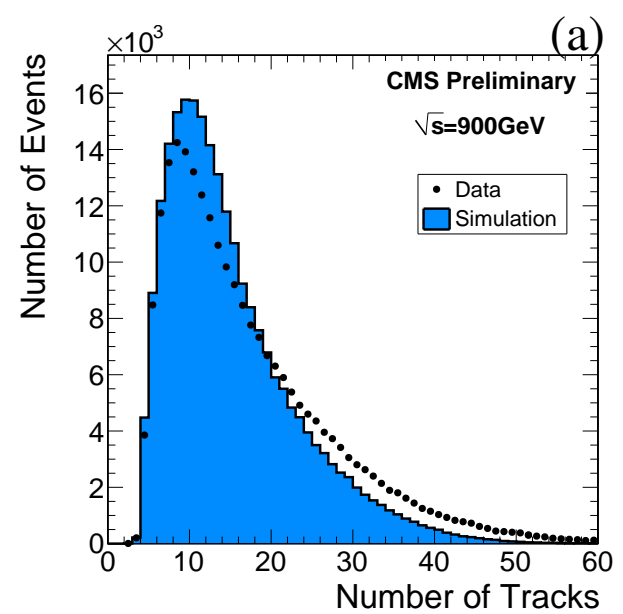

(c)

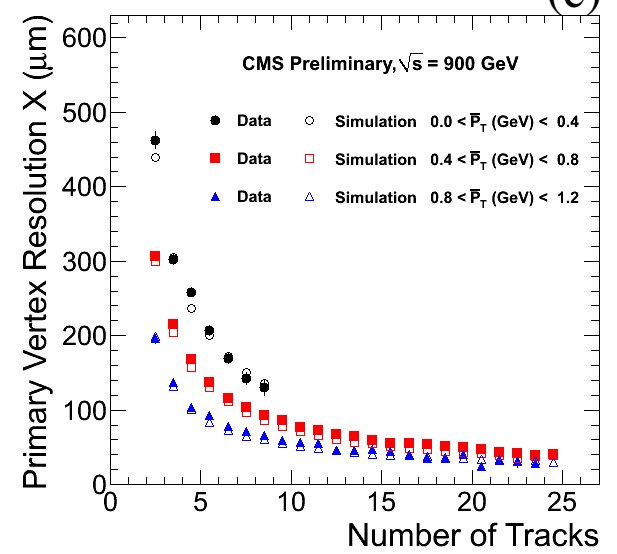

(b)

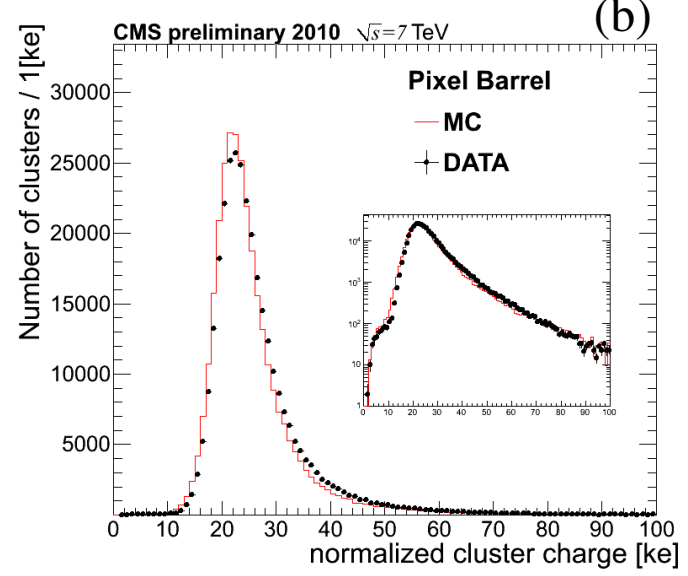

(d)

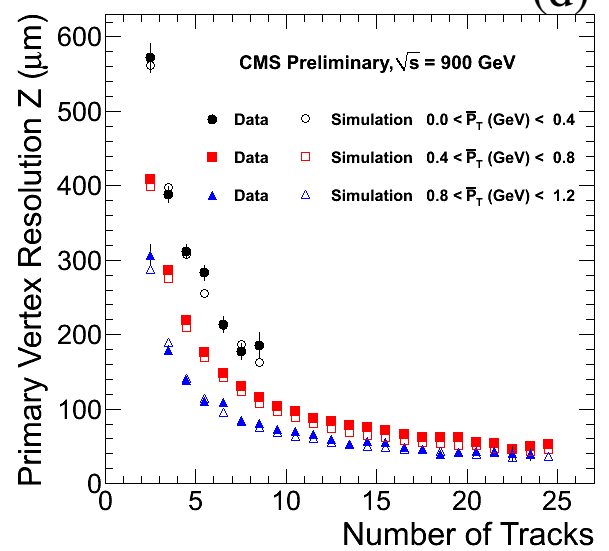

Figure 1: Example tracking performance plots, obtained in initial proton proton collision data at varying center of mass energies. (a) Track multiplicity, (b) barrel pixel cluster charge, normalized to a sensor thickness of $280 \mu \mathrm{m}$. Primary vertex resolution vs the number of tracks associated to the primary vertex: (c) in the transverse plane, (d) along the beam line.

the two tracks was required to be $d(K, \pi)<0.025 \mathrm{~mm}$. A vertex fit of the two tracks was performed and a significant displacement from the primary vertex of $3<l_{x y} / \sigma\left(l_{x y}\right)<20$ with a maximum error of $\sigma\left(l_{x y}\right)<0.03 \mathrm{~cm}$ was required. An important selection criterion consisted in the pointing angle $\alpha<0.1$, where $\alpha$ is the angle between the $D^{0}$ momentum and the separation between the primary vertex and the $D^{0}$ decay vertex. The resulting $D^{0}$ signal is displayed in Fig. 4(a) and shows a signal width of $16 \pm 2 \mathrm{MeV}$.

In a dataset of 37 million minimum bias events the decay channel $D^{*+} \rightarrow D^{0} \pi_{s}^{+}$with the subsequent decay $D^{0} \rightarrow K^{-} \pi^{+}$was reconstructed. The kinematic selection of this channel consisted in $p_{\perp}\left(\pi_{s}\right)>0.25 \mathrm{GeV}, p_{\perp}(\pi, K)>0.6 \mathrm{GeV}$, and $p_{\perp}\left(D^{*+}\right)>5 \mathrm{GeV}$. The distribution of the mass difference $m\left(K \pi \pi_{s}\right)-m(K \pi)$ is shown in Fig. 4(b). The resolution amounts to about $700 \mathrm{keV}$, where no fit constraints have been applied. With a mass selection of $\left|m_{K \pi \pi_{s}}-m_{K \pi}-\delta m_{\mathrm{PDG}}\right|<1.2 \mathrm{MeV}$ a clear $D^{0}$ signal is observable (not shown). 
(a)

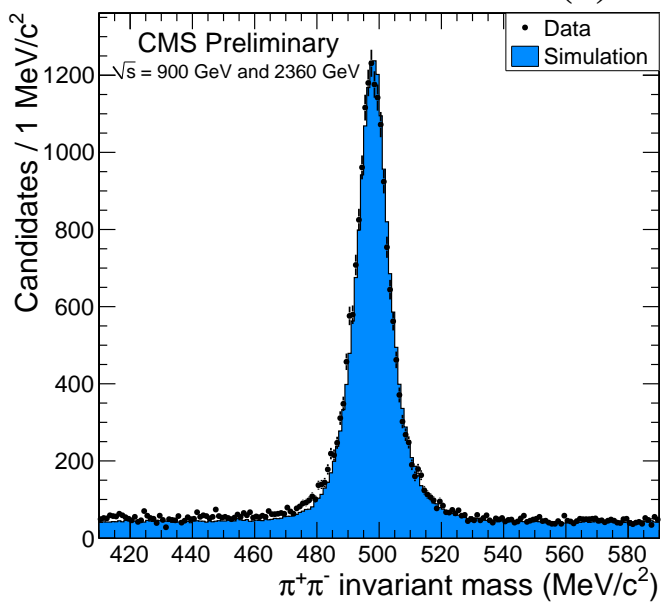

(b)

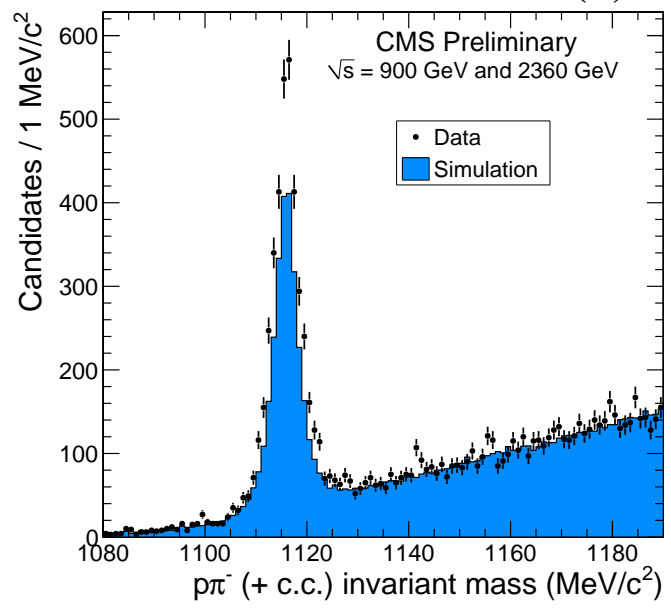

Figure 2: Reconstruction of long-lived resonances: (a) $K_{S}^{0} \rightarrow \pi^{+} \pi^{-}$and (b) $\Lambda \rightarrow p \pi^{-}$. The MC simulated event sample is normalized to the reconstructed $K_{S}^{0}$ yield. The production of strange baryons is underestimated in the MC simulation.

(a)

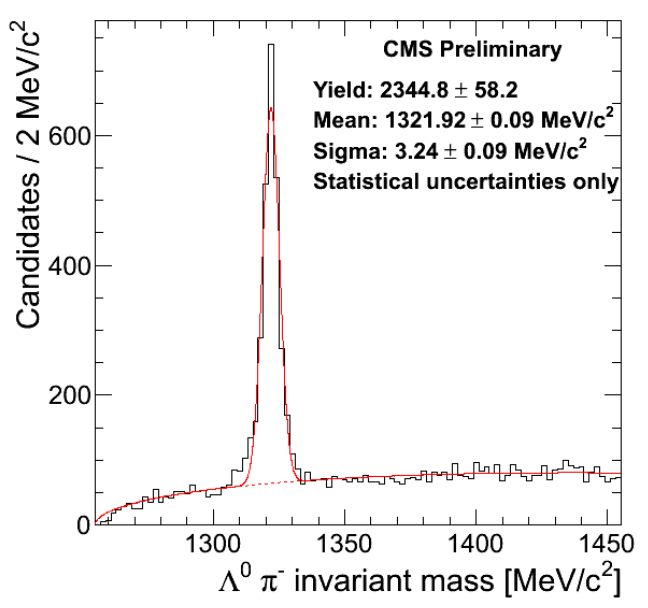

(b)

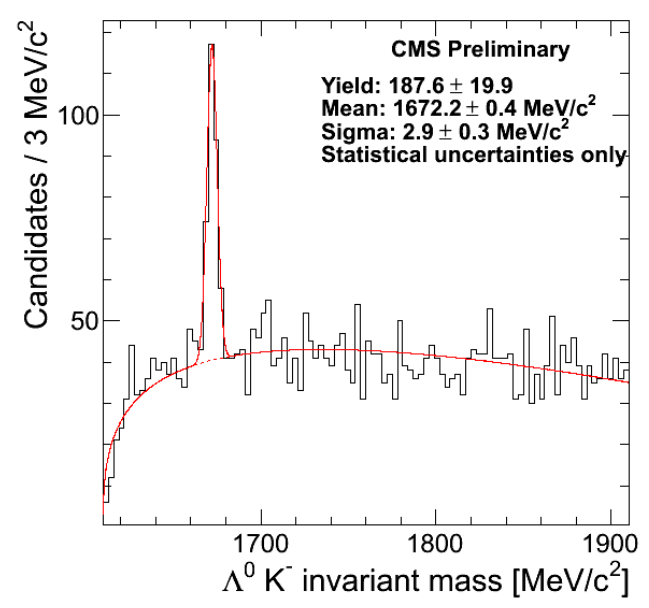

Figure 3: Reconstruction of heavy baryons: (a) $\Xi^{-} \rightarrow \Lambda \pi^{-}$and (b) $\Omega^{-} \rightarrow \Lambda K^{-}$.

The exclusive reconstruction of $D^{+} \rightarrow K^{-} \pi^{+} \pi^{+}$used a dataset of approximately 11 million minimum bias events. A very loose transverse momentum selection of $p_{\perp}>0.1 \mathrm{GeV}$ is complemented by a momentum requirement of $p>1 \mathrm{GeV}$. The vertex requirements include a pointing criterion $(5 \sigma)$ and a flight length significance of $l / \sigma(l)>7$. The resulting mass distribution is shown in Fig. 4(c).

\section{4. $J / \psi$ Signal}

A substantial effort is underway in CMS to measure the differential charmonium production 
(a)

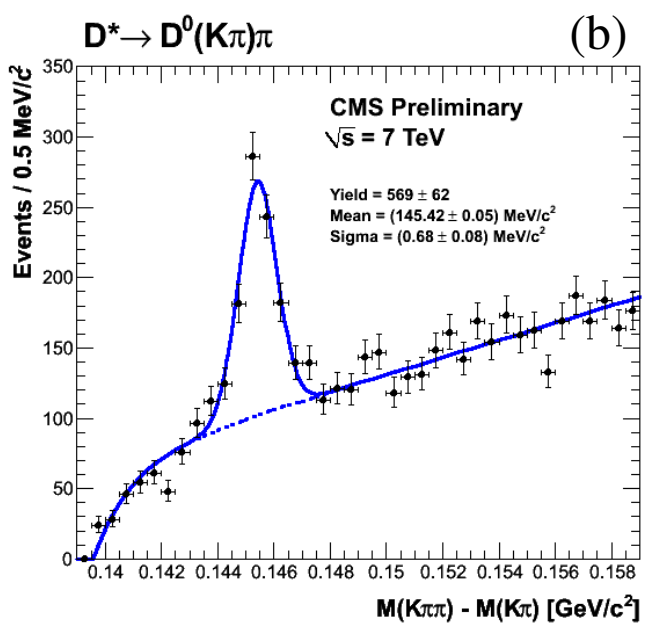

(c)

(d)
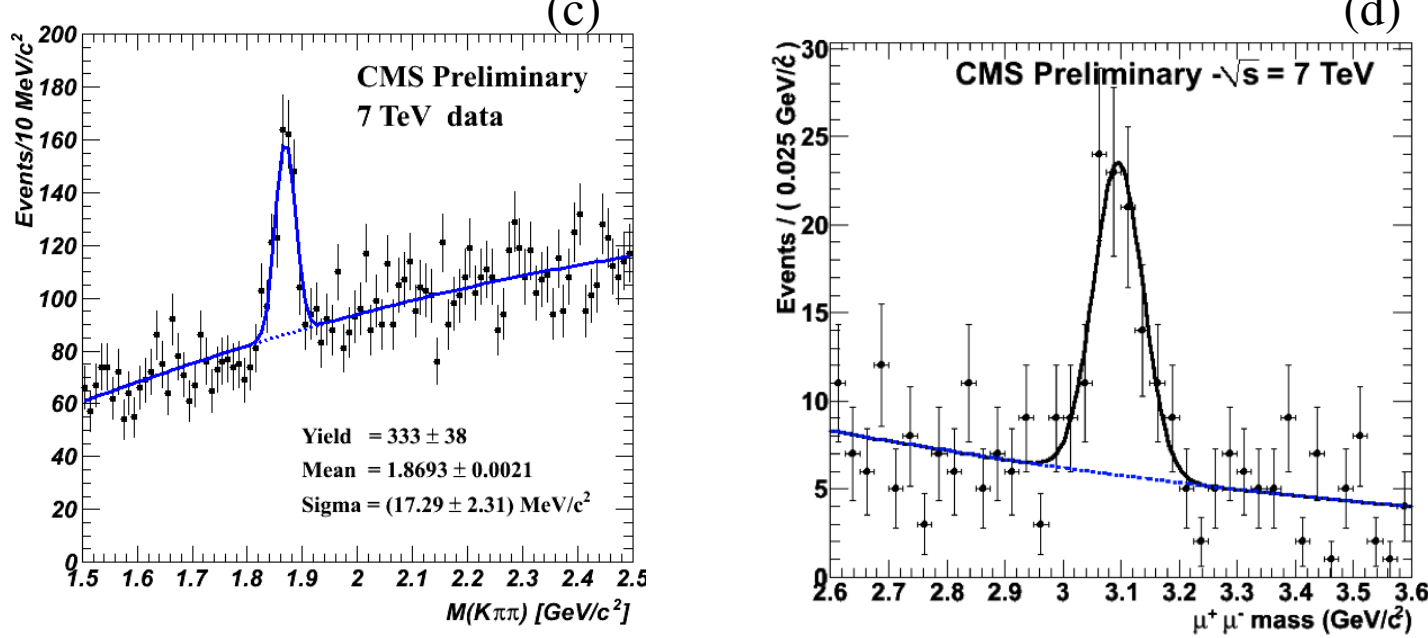

Figure 4: Exclusive reconstruction of open and hidden charm resonances. (a) invariant mass of $D^{0} \rightarrow K^{-} \pi^{+}$

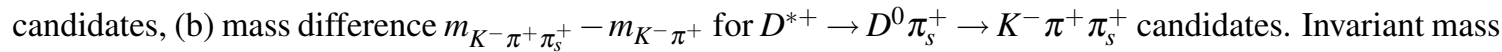
for (c) $D^{+} \rightarrow K^{-} \pi^{+} \pi^{+}$and (d) $J / \psi \rightarrow \mu^{+} \mu^{-}$candidates.

cross section and polarization. While this subject does not pertain to "flavor physics" in the strict sense, it is an essential prerequisite for a quantitative background understanding. Furthermore, dimuons are a prominent part of many final states in the heavy flavor program of CMS.

The decay $J / \psi \rightarrow \mu^{+} \mu^{-}$was reconstructed in a dataset corresponding to an integrated luminosity $\mathscr{L} \approx 1 \mathrm{nb}^{-1}$, triggered with a single-muon trigger with a transverse momentum threshold of $p_{\perp}>3 \mathrm{GeV}$. Muons were identified as (1) global muons and/or (2) tracker muons. The muon tracks measured in the inner tracker were required to have more than 10 hits and impact parameters very loosely consistent with the primary vertex $\left(d_{z}<20 \mathrm{~cm}\right.$ and $\left.d_{0}<5 \mathrm{~cm}\right)$. The two tracks were fit to a common vertex and its probability was required to be $P\left(\chi^{2}\right)>0.1 \%$. The resulting yield is illustrated in Fig. 4(d), where global muons were combined with tracker muons. The observed mass resolution is a strong function of the muon pseudorapidity: the best resolution is obtained in 
the barrel part of the detector where the magnetic field is very homogeneous.

\section{5. $B_{s}^{0} \rightarrow \mu^{+} \mu^{-}$Sensitivity}

The leptonic decay $B_{s}^{0} \rightarrow \mu^{+} \mu^{-}$provides excellent sensitivity to models of new physics with extended Higgs boson sectors. Such models include generic type-II Higgs doublet models or, for example supersymmetry, especially models with large $\tan \beta$ values. In the Standard Model (SM), the branching fraction is $\mathscr{B}\left(B_{s}^{0} \rightarrow \mu^{+} \mu^{-}\right)=(3.86 \pm 0.15) \times 10^{-9}$ [6]. In the SM, the decay $B_{s}^{0} \rightarrow \mu^{+} \mu^{-}$is Cabibbo-favored compared to $B^{0} \rightarrow \mu^{+} \mu^{-}$, and this is also the case in new physics models with minimal-flavor violation.

The background in this analysis [7] is dominated by two independent semileptonic $B$ decays and by the combination of one muon from a semileptonic $B$ decay with a hadron misidentified as a muon (either through in-flight decay or punch-through). Rare $B$ decays provide another important background contribution. The analysis requires a very strong background reduction while retaining a sufficiently high signal efficiency. This is achieved with a vertex fit of the two muons, requiring a significant flight length separation from the primary vertex $\left[l_{3 d} / \sigma\left(l_{3 d}\right)>17\right]$, and a pointing angle requirement of $\cos \alpha>0.9985$ (equivalent to $3.1^{\circ}$ ). Stringent isolation requirements reduce the background where the two muons arise from two independent $b$ hadrons, produced in gluon splitting. The isolation $I$ is defined as the ratio of

$$
I=\frac{p_{\perp}\left(\mu^{+} \mu^{-}\right)}{p_{\perp}\left(\mu^{+} \mu^{-}\right)+\Sigma_{t r k}\left|p_{\perp}\right|}
$$

where the sum runs over all tracks with $p_{\perp}>0.9 \mathrm{GeV}$ inside a cone with radius $\Delta R<1$ with respect to the $B_{s}^{0}$ candidate. A requirement of $I>0.85$ is applied.

To reduce the uncertainty from the luminosity and the unmeasured $B_{s}^{0}$ production cross section, the signal yield is normalized to the yield of $B^{+} \rightarrow J / \psi K^{+}$, where only the two muons are used in the $B$ decay vertex reconstruction and the selection has been tuned to minimize the differences to the signal reconstruction efficiency. With an integrated luminosity of $1 \mathrm{fb}^{-1}$ at $\sqrt{s}=14 \mathrm{TeV}$ it is expected to obtain an upper limit of $1.6 \times 10^{-8}$ at $90 \%$ C.L. This is a substantial improvement over the previous CMS result. No pile-up events were included in this simulation study. Improvements are possible by including tracker muons, the much more open trigger budget foreseen in the first years of LHC operation, and more sophisticated analysis methods.

\section{Top quark flavor physics}

The LHC at a center of mass energy of $\sqrt{s}=14 \mathrm{TeV}$ will be the first top factory (in a very similar sense to the $B$ factories of the past decade) and therefore will push the study of top flavor physics into a new regime. The production cross section for top quark pairs amounts to $\sigma(p p \rightarrow t \bar{t}) \approx 850 \mathrm{pb}$ at the next to leading order, with roughly a $10 \%$ error from scale and parton distribution function uncertainties. This presents an increase by a factor of about 100 with respect to the Tevatron, and will be bolstered additionally by roughly two orders of magnitude from the expected luminosity increase. Top quark events are categorized according to the decay of the accompanying $W$ as hadronic, semileptonic, or leptonic events. In $22 \%$ of all top quark decays, the charged lepton is a high- $p_{\perp}$ muon or electron. 
Since most top quark decays result in a $b$ quark, the identification of high- $p_{\perp} b$ quarks (or their jets) is very important. This is the subject of $b$ tagging (in the present context, this is not $b$ flavor tagging, i.e., whether a given neutral meson is a $B$ or $\bar{B}$ meson). The algorithms measure the track impact parameter with respect to the primary vertex, attempt to reconstruct (displaced) secondary vertices, or use leptons close to the jet. Algorithms combining several approaches often provide the highest $b$ tagging efficiency while keeping the mistag rate low. An efficiency $\varepsilon_{b} \approx 50 \%$ and a mistag rate of $\approx 0.1 \%$ constitute a good performance.

Arguably the most basic flavor measurement in top quark physics consists in the determination of the ratio [8]

$$
\begin{aligned}
R & =\frac{\Gamma(t \rightarrow b W)}{\Gamma(t \rightarrow q W)} \\
& =\frac{\left|V_{t b}\right|^{2}}{\left|V_{t d}\right|^{2}+\left|V_{t s}\right|^{2}+\left|V_{t b}\right|^{2}} \quad \text { (in the SM) }
\end{aligned}
$$

In the SM this observable constitutes a direct measurement of $\left|V_{t b}\right|$, in models with, e.g., four generations, $R$ provides constraints on $\left|V_{t b}\right|$.

The parameter $R$ is measured by counting the number of $b$ jets in semileptonic $t \bar{t}$ events. This number depends not only on $R$, but also on the $b$ tagging efficiency and the mistag probability. Therefore this analysis allows either a determination of $R$ or the $b$ tagging efficiency. The event selection is based on a high- $p_{\perp}$ lepton $\left(p_{\perp}>30 \mathrm{GeV}\right)$ and at least four jets with transverse energy $E_{T}>40 \mathrm{GeV}$. The background contamination is reduced with the centrality $C=$ $\Sigma E_{T} /\left(\sqrt{(\Sigma E)^{2}-\left(\Sigma p_{z}\right)^{2}}>0.35\right.$. With a luminosity of $250 \mathrm{pb}^{-1}$ at $\sqrt{s}=10 \mathrm{TeV}$, we expect to measure $R$ with a total error of $10 \%$, assuming that $\varepsilon_{b}$ is taken from an independent measurement. The dominant systematic error arises from the jet energy scale.

With substantially larger datasets (of the order $20-40 \mathrm{fb}^{-1}$ ), the search for FCNC top quark decays are probed at a level so far not accessible. The SM expectation is very low for these decays: $\mathscr{B}(t \rightarrow q g)=5 \times 10^{-11}, \mathscr{B}(t \rightarrow q \gamma)=5 \times 10^{-13}$, and $\mathscr{B}(t \rightarrow q Z)=\times 10^{-13}$. Various models provide expectations ranging up to $10^{-2}$ (partially already excluded by measurements at HERA, LEP2, and Tevatron). The expected results at CMS will allow limits at the few times $10^{-4}$ level for the channels with a photon or $Z$ boson in the final state.

\section{Conclusions}

The data collection at CMS has started very successfully in fall 2009 with proton proton collisions at center of mass energies of $\sqrt{s}=0.9,2.36$, and $7 \mathrm{TeV}$. A multitude of light and heavy particles has been reconstructed as expected. The masses are in agreement with the known world averages, and the observed widths are consistent with the MC simulation. The muon triggers are operating at very low thresholds allowing for a detailed investigation of physics with one or two muons among the final state particles.

In summer 2010, the study of heavy flavor physics in CMS will focus on the measurement of heavy flavor production. This includes the differential production cross sections of quarkonia $(J / \psi$ and $\Upsilon$ ), and the inclusive measurement of open $b$ quark production. The study of $b \bar{b}$ quark 
correlations and the $t \bar{t}$ production cross section will follow. With an integrated luminosity of more than a few $100 \mathrm{pb}^{-1}$ 'proper' heavy flavor physics results in $B_{s}^{0}$ decays can be expected: The study of $B_{s}^{0} \rightarrow J / \psi \phi$ decays will measure the decay width difference $\Delta \Gamma_{s}$ and the search for the very rare decay $B_{s}^{0} \rightarrow \mu^{+} \mu^{-}$provides sensitivity to new physics models with extended Higgs boson sectors.

Ultimately, the integrated luminosity will allow the observation of the very rare decays $B_{s}^{0} \rightarrow$ $\mu^{+} \mu^{-}$and $B^{0} \rightarrow \mu^{+} \mu^{-}$. Rare top quark decays mediated by flavor changing neutral currents will provide a completely new set of constraints on new physics models. A very rich field of heavy flavor physics is waiting to be harvested at CMS.

\section{References}

[1] CMS Collaboration, R. Adolphi et. al., The CMS experiment at the CERN LHC, JINST 0803 (2008) S08004.

[2] CMS Collaboration, S. Chatrchyan et. al., Performance of CMS Muon Reconstruction in Cosmic-Ray Events, JINST 5 (2010) T03022.

[3] T. Sjostrand, S. Mrenna, and P. Z. Skands, PYTHIA 6.4 Physics and Manual, JHEP 05 (2006) 026.

[4] CMS Collaboration, R. Adolphi et. al., CMS Tracking Performance Results from Early LHC Operation, CERN-PH-EP-2010-019; submitted to the European Physical Journal C (2010).

[5] CMS Collaboration, Public webpage with CMS Tracking Results, https://twiki.cern.ch/twiki/bin/view/CMSPublic/PhysicsResultsTRK.

[6] M. Artuso et. al., B, D and K decays, Eur. Phys. J. C57 (2008) 309-492, [arXiv: 0801 . 1833].

[7] CMS Collaboration, R. Adolphi et. al., Study of $B_{s}$ Mesons Decaying to $\mu \mu$ in CMS,

[8] CMS Collaboration, R. Adolphi et. al., Plan for a $\mathscr{B}(t \rightarrow W b) / \mathscr{B}(t \rightarrow W q)$ measurement in $t \bar{t}$ semi-leptonic decays at $\sqrt{s}=10 \mathrm{TeV}$. 